\title{
LỰA CHỌN PHƯƠNG PHÁP TÍNH SAI SỐ TRUNG PHƯƠNG VỊ TRÍ ĐIỂM GIỮA PHỤC VỤ VIỆC ĐÁNH GIÁ ĐỘ TIN CẬY CỦA ĐƯờNG CHUYỀN ĐO HAI GÓC NỐI
}

\author{
PGS. TS. TRƯƠNG QUANG HIÉU(1), ThS. TÓNG TH! HẠNH ${ }^{(2)}$ \\ (1)Trường Đại học Mỏ - Địa chất \\ ${ }^{(2) H o ̣ c ~ v i e ̣ ̂ n ~ K y ̃ ~ t h u a ̣ ̂ t ~ Q u a ̂ n ~ s u ̛ ̣ ~}$
}

\section{Tóm tắt:}

Bài báo nhằm phân tích khả năng ứng dụng của sai số trung phương vị trí điểm giữa để đánh giá độ tin cậy đường chuyền đo hai góc nối. Đề xuất các phương pháp tính sai số vị trí điểm giữa, từ đó lựa chọn phương pháp đánh giá độ chính xác hợp lý.

\section{1. Đặt vấn đề}

Để phương án đo:

$$
W:=<L_{W}, X_{W}, U_{W}>
$$

là đường chuyền đo 2 góc nối có $p$ điểm cần tìm thì:

- Vector trị đo $L_{W}$ là vector gồm $(p+2)$ góc và $(p+1)$ cạnh đo.

- Vector đại lượng cần tìm $X_{W}$ là vector tọa độ của $p$ điểm cần tìm.

- Các ràng buộc $U_{W}$ có thể là ràng buộc giữa các điểm gốc và các điểm cần tìm tạo thành một đường đa giác.

Mục đích cuối cùng của phương án đo (1.1) là xác định tọa độ $p$ điểm cần tìm với độ tin cậy cao nhất. Độ tin cậy trên đặc trưng bằng ma trận hiệp phương sai:

$$
\mathrm{M}_{\mathrm{X}_{\mathrm{W}}^{*}}=\sigma_{0}^{2} \cdot \mathrm{Q}_{\mathrm{Xx}}(2 \mathrm{p} \times 2 \mathrm{p})
$$

Lựa chọn các "đặc số" đặc trưng cho ma trận (1.2) là hàm mục tiêu đánh giá độ tin cậy của đường chuyền đo 2 góc nối phải có tính khoa học và thực tiễn. Trong bài báo này chúng tôi chọn một dạng hàm mục tiêu là sai số trung phương vị trí điểm giữa của đường chuyền. Hàm mục tiêu này là một dạng đơn giản của vết ma trận (1.2) khi không xét đến ảnh hưởng của sai số vị trí các điểm cần tìm còn lại trong đường chuyền. Sau khi phân tích khả năng đặc trưng của hàm mục tiêu trên, để đánh giá độ tin cậy của đường chuyền chúng tôi sẽ đưa ra một số phương pháp tính sai số trung phương vị trí điểm giữa nhằm tăng khả năng ứng dụng của hàm mục tiêu này.

\section{Nội dung}

Sai số trung phương vị trí điểm giữa (điểm yếu) của đường chuyền đo 2 góc nối tính theo công thức:

$$
\mathrm{M}_{\mathrm{G}}^{2}=\mathrm{M}_{\mathrm{X}_{\mathrm{G}}}^{2}+\mathrm{M}_{\mathrm{Y}_{\mathrm{G}}}^{2}=\sigma_{0}^{2} \cdot\left(\mathrm{Q}_{\mathrm{XX}_{\mathrm{G}}}+\mathrm{Q}_{\mathrm{YY}_{\mathrm{G}}}\right)
$$


Đẳng thức (1.3) là một dạng "đặc số" đơn giản của ma trận (1.2). Vì tổng bình phương sai số trung phương vị trí điểm tính theo hai hướng tương ứng vuông góc bất kỳ là một hằng số, nên khi $\mathrm{M}_{\mathrm{ng}_{\mathrm{G}}}$ và $\mathrm{M}_{\mathrm{d}_{\mathrm{G}}}$ là sai số dịch vị ngang và dịch vị dọc của điểm giữa $\mathrm{G}$ thì ta viết được:

$$
\mathrm{M}_{\mathrm{G}}^{2}=\mathrm{M}_{\mathrm{X}_{(\mathrm{G})}}^{2}+\mathrm{M}_{\mathrm{Y}_{(\mathrm{G})}}^{2}=\mathrm{M}_{\mathrm{ng}(\mathrm{G})}^{2}+\mathrm{M}_{\mathrm{d}(\mathrm{G})}^{2}=\text { const }
$$

Để sử dụng vế sau của công thức (1.4), GS. Hausbrandt [1] đã chứng minh được công thức tính sai số trung phương dịch vị dọc, dịch vị ngang điểm thứ $\mathrm{i}(\mathrm{i}=1 \div \mathrm{p})$ của đường chuyền lý tưởng (duỗi thẳng, cạnh đều) đo 2 góc nối, cụ thể:

- Sai số dịch vị ngang:

$$
\begin{aligned}
\mathrm{M}_{\mathrm{ng}_{(\mathrm{i})}} & =\frac{\sigma_{\beta}}{\rho^{\prime \prime}} \cdot S \cdot \sqrt{\frac{\mathrm{i}(\mathrm{p}-\mathrm{i}+1)(\mathrm{p}-\mathrm{i}+2)(\mathrm{i}+1)}{6(\mathrm{p}+1)(\mathrm{p}+2)(\mathrm{p}+3)}\left[\mathrm{p}(2 \mathrm{i}+1)-\left(2 \mathrm{i}^{2}-2 \mathrm{i}-3\right)\right]} \\
& =\frac{\sigma_{\beta}}{\rho^{\prime \prime}} \cdot \operatorname{S.M} \overline{\mathrm{M}}_{\mathrm{ng}_{(\mathrm{i})}}
\end{aligned}
$$

- Sai số dịch vị dọc:

$$
M_{d_{(i)}}=\sigma_{S} \sqrt{\frac{i(p-i+1)}{p+1}}=\sigma_{S} \cdot \bar{M}_{d_{(i)}}
$$

Khi: $\quad \frac{\sigma_{\beta}}{\rho^{\prime \prime}} \approx \frac{\sigma_{S}}{S}$

Hay: $\quad \mathrm{u}=\frac{\mathrm{T}_{S}}{\mathrm{~T}_{\beta}} \approx 1$

với: $\sigma_{S}$ và $\sigma_{\beta}$ là sai số trung phương của cạnh đo và góc đo,

$T_{S}$ và $T_{\beta}$ là mẫu số của sai số trung phương tương đối dãy trị đo góc và dãy trị đo cạnh.

Thì các sai số $\mathrm{M}_{\mathrm{ng}_{(\mathrm{i})}}$ và $\mathrm{M}_{\mathrm{d}_{(\mathrm{i})}}$ chỉ phụ thuộc vào số lượng điểm cần tìm $\mathrm{p}$ và chỉ số của điểm cần tìm trong đường chuyền. Đồng thời ta có tỷ số:

$$
\frac{\mathrm{M}_{\mathrm{ng}_{(\mathrm{i})}}}{\mathrm{M}_{\mathrm{d}_{(\mathrm{i})}}} \approx \frac{\overline{\mathrm{M}}_{\mathrm{ng}_{(\mathrm{i})}}}{\overline{\mathrm{M}}_{\mathrm{d}_{(\mathrm{i})}}}
$$

Trong bảng (1-1) là giá trị và sự thay đổi các giá trị $\overline{\mathrm{M}}_{\mathrm{ng}}$ và $\overline{\mathrm{M}}_{\mathrm{d}_{(\mathrm{i})}}$ với số điểm cần tìm $p=7$ điểm và $p=9$ điểm. (Xem bảng 1 )

Từ kết quả trong bảng 1-1, ta thấy:

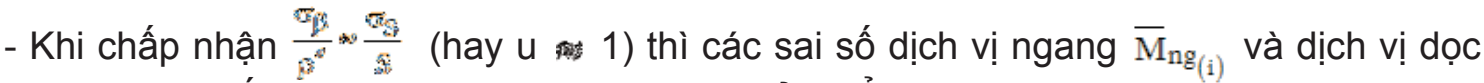
$\overline{\mathrm{M}}_{\mathrm{d}_{(\mathrm{i})}}$ có giá trị đối xứng qua giá trị tương ứng của điểm giữa.

- Tại điểm giữa của đường chuyền các sai số $\overline{\mathrm{M}}_{\mathrm{ng}(\mathrm{i})}$ và $\overline{\mathrm{M}}_{\mathrm{d}_{(\mathrm{i})}}$ có giá trị lớn nhất, các giá trị tăng dần khi số lượng điểm $p$ cần tìm tăng. 
Bảng (1-1)

Giá trị $\bar{M}_{n g_{(i)}}$ và $\bar{M}_{d_{(i)}}$ với các trường họ̣p $p=7$ điểm và $p=9$ điểm

\begin{tabular}{|c|c|c|c|c|c|c|c|c|c|c|}
\hline $\begin{array}{c}\text { Số } \\
\text { lưởng } \\
\text { điếm }\end{array}$ & $\begin{array}{c}\overline{\mathrm{M}}_{\mathrm{ng}} \\
\overline{\mathrm{M}}_{\mathrm{d}_{(\mathrm{i})}}\end{array}$ & 1 & 2 & 3 & 4 & 5 & 6 & 7 & 8 & 9 \\
\hline 7 & $\overline{\mathrm{M}}_{\mathrm{ng}}$ & 0,789 & 1,408 & 1,826 & 1,972 & 1,826 & 1,408 & 0,789 & & \\
\cline { 2 - 12 } & $\overline{\mathrm{M}}_{\mathrm{d}_{(\mathrm{i})}}$ & 0,935 & 1,225 & 1,369 & 1,414 & 1,369 & 1,225 & 0,935 & & \\
\hline \multirow{2}{*}{9} & $\overline{\mathrm{M}}_{\mathrm{ng}}$ & 0,826 & 1,549 & 2,141 & 2,523 & 2,654 & 2,523 & 2,141 & 1,549 & 0,826 \\
\cline { 2 - 11 } & $\overline{\mathrm{M}}_{\mathrm{d}_{(\mathrm{i})}}$ & 0,949 & 1,265 & 1,449 & 1,549 & 1,581 & 1,549 & 1,449 & 1,265 & 0,949 \\
\hline
\end{tabular}

- Trừ các điểm đầu và điểm cuối của đường chuyền thì các sai số dịch vị ngang luôn lớn hơn sai số dịch vị dọc. Độ lệch này sẽ đạt giá trị lớn nhất tại vị trí điểm giữa. Khi số lượng điểm cần tìm tăng thì độ lệch này cũng tăng.

Từ các phân tích trên cho phép ta sử dụng sai số trung phương vị trí điểm giữa làm hàm mục tiêu đánh giá độ tin cậy của đường chuyền đo 2 góc nối. Dùng hàm mục tiêu này để so sánh với các chỉ tiêu kỹ thuật tương ứng.

Các công thức (1.5) được chứng minh chặt chẽ bằng phương pháp bình sai gián tiếp đường chuyền lý tưởng đo 2 góc nối. Trong [2], PGS. Trương Quang Hiếu đã đăng tải các công thức tính sai số trung phương dịch vị ngang và dịch vị dọc của điểm giữa đường chuyền đo 2 góc nối, các công thức có dạng:

- Sai số dịch vị ngang:

$$
\mathrm{M}_{\mathrm{ng}(\mathrm{G})}=0,3536 \cdot \mathrm{L}_{1} \cdot \sqrt{\frac{\mathrm{N}+1,5}{3}} \cdot \frac{\sigma_{\beta}}{\rho^{\prime \prime}}
$$

- Sai số dịch vị dọc:

$$
\mathrm{M}_{\mathrm{d}_{(\mathrm{G})}}=0,7071 \cdot \sqrt{\mathrm{N}} \cdot \sigma_{\mathrm{S}}
$$

Trong đó: $\mathrm{N}=\frac{\mathrm{p}+1}{2} ; \mathrm{L}_{1}=\mathrm{N} . \mathrm{S} ; \mathrm{S}$ là chiều dài trung bình cạnh đường chuyền.

Từ các công thức (1.6a) và (1.6b) ta rút ra quan hệ giữa sai số dịch vị ngang và dịch vị dọc của điểm giữa đường chuyền:

$$
\frac{\mathrm{M}_{\mathrm{ng}_{(\mathrm{G})}}}{\mathrm{M}_{\mathrm{d}_{(\mathrm{G})}}} \approx \frac{1}{2} \cdot \mathrm{u} \cdot \sqrt{\frac{\mathrm{N}(\mathrm{N}+1,5)}{3}}
$$


Tỷ số (1.7) chủ yếu phụ thuộc vào lượng điểm cần tìm trong đường chuyền. Cụ thể, khi $\mathrm{u}$ 1, với đường chuyền có $\mathrm{p}=7$ điểm cần tìm thì $\frac{\mathrm{M}_{\mathrm{ng}_{(\mathrm{G})}}}{\mathrm{M}_{\mathrm{M}_{(\mathrm{G})}}} \approx 1,35$ và với đường chuyền có $\mathrm{p}$ $=9$ điểm cần tìm thì $\frac{\mathrm{M}_{\mathrm{ng}_{(G)}}}{\mathrm{M}_{\mathrm{d}_{(G)}}} \approx 1,65$. Các tỷ số này có giá trị gần bằng các tỷ số tương ứng khi tính theo công thức $(1.5 \mathrm{C})$ (theo công thức $1.5 \mathrm{c}$, với $\mathrm{p}=7$ thì $\frac{\mathrm{M}_{\mathrm{n}_{(G)}}}{\mathrm{M}_{\mathrm{n}_{(G)}}} \approx 1,39$ và $\mathrm{p}=9$ thì $\frac{\mathrm{M}_{\mathrm{n}_{(G)}}}{\mathrm{M}_{\mathrm{d}_{(\mathrm{G})}}} \approx 1,68$. .

Vậy để tính sai số trung phương vị trí điểm giữa đường chuyền đo 2 góc nối chúng ta có thể lựa chọn một trong các phương pháp sau đây:

- Tính sai số trung phương vị trí điểm giữa theo công thức (1.3), trong đó

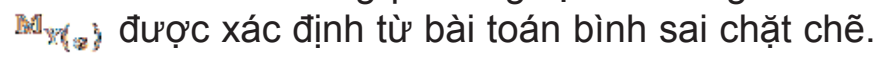

Tính sai số trung phương vị trí điểm giữa theo các công thức (1.6a) và (1.6b) khi biết sai số trung phương dãy trị đo góc $\left(\sigma_{\beta}^{*}\right)$ và dãy trị đo cạnh $\left(\sigma_{\mathrm{S}}^{*}\right)$ sau bình sai. Khi sử dụng các công thức này với trường hợp đường chuyền có cạnh không đều nhau thì đại lượng $\mathrm{L}_{1}$ được tính từ cạnh khởi tính đến điểm giữa.

Tính sai số trung phương vị trí điểm giữa dựa vào việc tính sai số trung phương hoặc của dãy trị đo góc $\sigma_{\beta}^{*}$ hoặc của dãy trị đo cạnh $\sigma_{S}^{*}$. và quan hệ (1.7) khi nhận u 1 . Thông

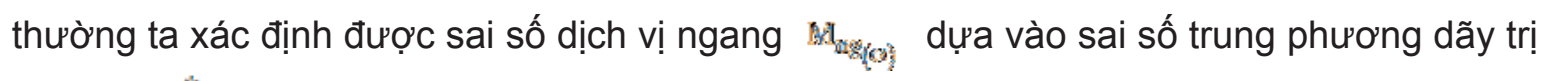
đo góc $\left(\sigma_{\beta}^{*}\right)$ bằng cách nhận trọng số $P=1$. Sau bình sai sai số trung phương trọng số đơn vị $\sigma_{0}=\sigma_{\beta}^{*}$. Phương pháp này sẽ cho giá trị gần đúng của sai số trung phương vị trí điểm giữa vì nhận u 1 .

\section{Tính toán thực nghiệm}

Nội dung của thực nghiệm là sử dụng các phương pháp trình bày trong bài báo để tính sai số trung phương vị trí điểm giữa của đường chuyền địa chính đo 2 góc nối trên hình 11. Đường chuyền có yêu cầu về sai số trung phương vị trí điểm là $\pm 5 \mathrm{~cm}$.

\subsection{Sơ đồ lưới}

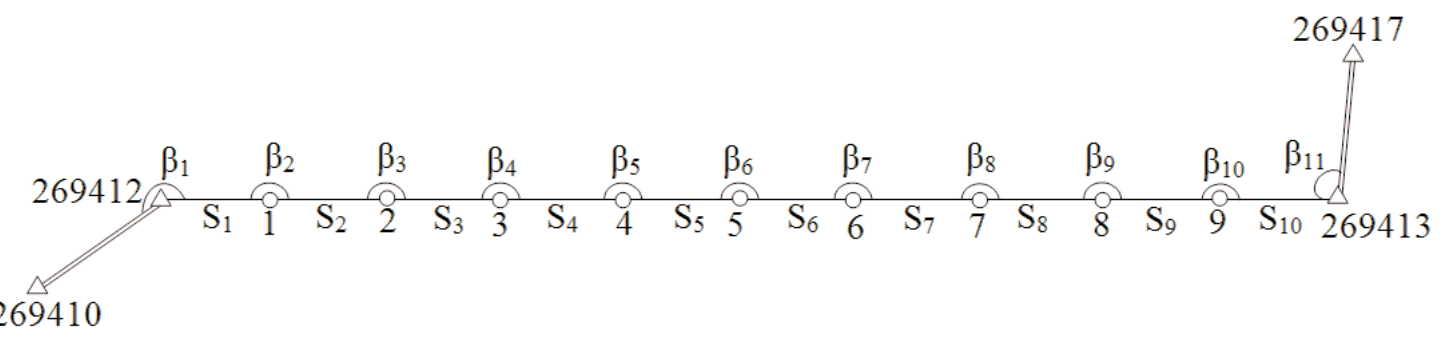

Hinh 1-1

\subsection{Số liệu gốc}


Nghiên cúu - Úng dụng

Bảng 1-2

\begin{tabular}{|c|c|c|}
\hline Tên điểm & $\mathbf{X}(\mathbf{m})$ & $\mathbf{Y}(\mathbf{m})$ \\
\hline $\mathbf{2 6 9 4 1 0}$ & 2011136,642 & 558395,440 \\
\hline $\mathbf{2 6 9 4 1 2}$ & 2009312,002 & 562701,304 \\
\hline $\mathbf{2 6 9 4 1 3}$ & 2004176,722 & 571078,531 \\
\hline $\mathbf{2 6 9 4 1 7}$ & 2001027,024 & 571206,792 \\
\hline
\end{tabular}

Bảng 1-3

\begin{tabular}{|c|ccc|c|c|}
\hline \multicolumn{5}{|c|}{ Trị đo góc } & \multicolumn{2}{c|}{ Trị đo cạnh } \\
\hline TT & $\left(^{0}\right)$ & $\left(^{\prime}\right)$ & $\left({ }^{\prime}\right)$ & TT & $(\mathrm{m})$ \\
\hline 1 & 179 & 06 & 13 & 1 & 1103,785 \\
\hline 2 & 205 & 28 & 56 & 2 & 1056,895 \\
\hline 3 & 147 & 52 & 50 & 3 & 1061,166 \\
\hline 4 & 204 & 10 & 02 & 4 & 965,077 \\
\hline 5 & 160 & 20 & 50 & 5 & 955,752 \\
\hline 6 & 201 & 32 & 18 & 6 & 938,771 \\
\hline 7 & 162 & 00 & 56 & 7 & 935,645 \\
\hline 8 & 191 & 12 & 49 & 8 & 1015,202 \\
\hline 9 & 188 & 37 & 47 & 9 & 984,481 \\
\hline 10 & 165 & 05 & 51 & 10 & 982,985 \\
\hline 11 & 239 & 13 & 26 & 11 & \\
\hline
\end{tabular}

\subsection{Tính sai số trung phương vị trí điểm giữa (điểm 5)}

Lưới (1-1) được bình sai chặt chẽ theo phương pháp điều kiện. Kết quả tính toán đầy đủ có trong [3], dưới đây chúng tôi chỉ trích những số liệu cần thiết phục vụ việc tính toán của bài báo.

Nhận trọng số các trị đo: $\mathrm{P}_{\beta}=\frac{1}{\sigma_{\beta}^{2}} ; \mathrm{P}_{\mathrm{S}}=\frac{1}{\sigma_{\mathrm{S}}^{2}}$

và sai số trung phương trọng số đơn vị sau bình sai: $\sigma_{0}^{*} \approx 1,0$

Trọng số đảo của tọa độ điểm 5 sau bình sai:

$$
\mathrm{Q}_{\mathrm{XX}}=0,126 ; \mathrm{Q}_{\mathrm{YY}} \mathrm{Y}_{(5)}=0,060
$$


Tổng số điểm cần tìm $p=9 ; \mathrm{N}=5 ; \mathrm{L}_{1}=5142,675 \mathrm{~m} ; \quad \bar{\sigma}_{\beta}^{*}=2,5^{\prime \prime} ; \bar{\sigma}_{\mathrm{S}}^{*}=1,0 \mathrm{~cm}$

Kết quả tính toán theo 3 phương pháp như sau:

1) Tính sai số trung phương vị trí điểm giữa từ kết quả bình sai chặt chẽ:

$$
M_{5}=\sigma_{0}^{*} \sqrt{Q_{X X_{(5)}}+Q_{Y Y_{(5)}}} \approx 0,43 \mathrm{dm}=4,3 \mathrm{~cm}
$$

2) Tính sai số trung phương vị trí điểm giữa dựa vào sai số dịch vị dọc, dịch vị ngang khi biết sai số trung phương dãy trị đo góc $\sigma_{\beta}^{*}$ và dãy trị đo cạnh $\sigma_{\mathrm{S}}^{*}$ :

$$
\begin{aligned}
& \mathrm{M}_{\mathrm{ng}_{(5)}}=0,3536 \cdot \mathrm{L}_{1} \cdot \sqrt{\frac{\mathrm{N}+1,5}{3}} \cdot \frac{\sigma_{\beta}^{*}}{\rho^{\prime \prime}} \approx 3,2 \mathrm{~cm} \\
& \mathrm{M}_{\mathrm{d}_{(5)}}=0,7071 \cdot \sqrt{\mathrm{N}} \cdot \sigma_{\mathrm{S}}^{*} \approx 1,6 \mathrm{~cm}
\end{aligned}
$$

Sai số vị trí điểm 5 sẽ là:

$$
\mathrm{M}_{5}=\sqrt{\mathrm{M}_{\mathrm{ng}(5)}^{2}+\mathrm{M}_{\mathrm{d}_{(5)}}^{2}}=\sqrt{3,2^{2}+1,6^{2}} \approx 3,6 \mathrm{~cm}
$$

3) Tính sai số trung phương vị trí điểm giữa dựa vào sai số dịch vị ngang khi chỉ biết sai

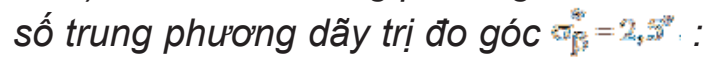

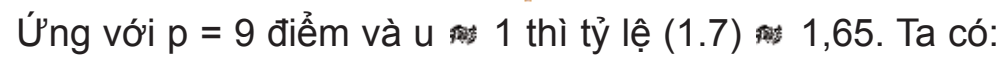

$$
\begin{aligned}
& \mathrm{M}_{\mathrm{ng}_{(5)}} \approx 3,2 \mathrm{~cm} \\
& \mathrm{M}_{\mathrm{d}_{(5)}}=\frac{\mathrm{M}_{\mathrm{ng}(5)}}{1,65} \approx 1,9 \mathrm{~cm}
\end{aligned}
$$

Cuối cùng:

$$
\mathrm{M}_{5}=\sqrt{\mathrm{M}_{\mathrm{ng}(5)}^{2}+\mathrm{M}_{\mathrm{d}_{(5)}}^{2}}=\sqrt{3,2^{2}+1,9^{2}}=3,7 \mathrm{~cm}
$$

\section{Kết luận}

Từ nội dung trình bày trong bài báo, chúng tôi rút ra một số kết luận sau:

- Dùng sai số trung phương vị trí điểm giữa có thể đánh giá độ tin cậy của đường chuyền đo 2 góc nối, vì chỉ cần đạt tiêu chuẩn kỹ thuật về sai số vị trí điểm giữa thì các điểm còn lại sẽ đạt chỉ tiêu về sai số vị trí điểm.

- Từ kết quả của ba cách tính sai số trung phương vị trí điểm trên ta thấy độ chênh giữa các kết quả là không lớn (cao nhất là $7 \mathrm{~mm}$ ). Độ chênh này được tạo nên chủ yếu từ tính 
chất lý tưởng của đường chuyền.

- Phương pháp tính thứ 3 được coi là phương pháp có tính hiệu quả nhất vì trong quá trình bình sai đường chuyền ta chỉ cần nhận trọng số $\mathrm{P}_{\beta}=1$ mà không cần phải lập một hàm trọng số nào nữa.O

\section{Tài liệu tham khảo}

[1]. S. Hausbrandt (1971), Rachunek, Wýrównawczy i obliczenia geodezyjne, P.P.W.K, Warszawa.

[2]. Trương Quang Hiếu (1988), projektowanie sieci geodezyjnych zaktadanych w warunkach pionierskich dta celów gospodarczych zeszczególnym uwzglsdnieniem Wietnamu, Praca doktorska, Warszawa.

[3]. Trương Minh Hùng (2016), Lựa chọn phương pháp xử lý tuyến đường chuyền phục vụ nghiên cứu biến dạng đập thủy điện, luận văn Thạc sỹ Kỹ thuật, Hà Nội.

[4]. Trương Quang Hiếu và Lê Ngọc Giang (2014), Xác định đại lượng đặc trưng độ tin cậy của đường chuyền, Tạp chí Khoa học Đo đạc và Bản đồ số 22.

[5]. Nguyễn Việt Hải Linh (2014), Đánh giá độ tin cậy đường chuyền thông qua đại lượng đặc trưng cho Elip sai số vị trí điểm, luận văn Thạc sỹ Kỹ thuật, Trường Đại học Mỏ - Địa chất, Hà Nội.O

\section{Summary}

Selection of method for calculating the standard deviation of midstation's position in order to evaluate the precision of closed connecting traverse

Assoc. Prof. Dr. Truong Quang Hieu, University of Mining and Geology

MSc. Tong Thi Hanh, Military Technical Academy

The paper aims to analyze the applicability of the standard deviation of midstation's position to evaluate the precision of closed connecting traverse. A suitable calculation method of the standard deviation of midstation's position is chosen from several shown ones. $O$ 